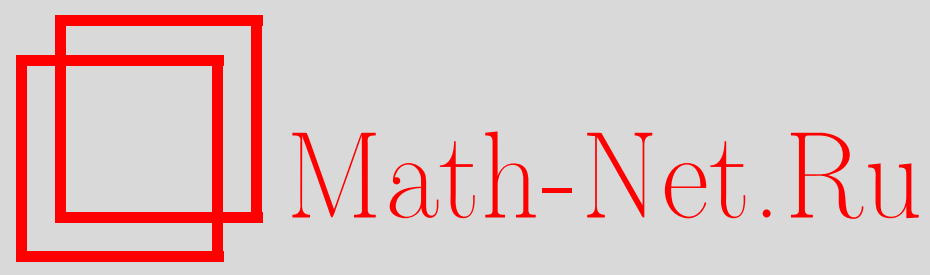

В. Г. Ламбурт, Сопряженные точки на геодезической со случайной кривизной, Матем. заметки, 2006, том 79, выпуск 1, 95-101

DOI: https://doi.org/10.4213/mzm2677

Использование Общероссийского математического портала Math-Net.Ru подразумевает, что вы прочитали и согласны с пользовательским соглашением http://www . mathnet.ru/rus/agreement

Параметры загрузки:

IP: 18.234 .156 .22

26 апреля 2023 г., $14: 18: 37$

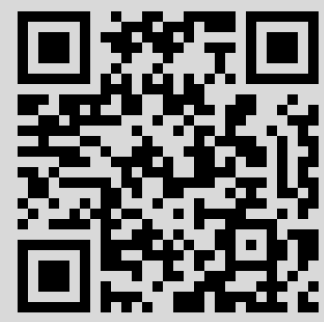




\title{
СОПРЯЖЕННЫЕ ТОЧКИ НА ГЕОДЕЗИЧЕСКОЙ СО СЛУЧАЙНОЙ КРИВИЗНОЙ
}

\author{
В.Г. Ламбурт
}

Изучаются сопряженные точки на обновляющейся геодезической, кривизна вдоль которой является случайным процессом. Приводится построение верхней оценки для среднего расстояния между соседними сопряженными точками.

Библиографияя: 4 названия.

1. Введение. Рассмотрение римановых многообразий со случайной кривизной предоставляет дополнительные возможности для выделения общих свойств различных групп римановых многообразий. Введение вероятностных представлений в риманову геометрию позволяет задавать меру на определенных классах римановых многообразий и получать свойства, справедливые, скажем, для почти всех многообразий.

Основные результаты геометрии в целом о строении римановых многообразий относятся либо к объектам с положительной, либо к объектам с отрицательной кривизной; например, теорема о сфере [1] или теорема Ефимова [2]. Между тем, хотелось бы получить какие-нибудь общие результаты о строении объектов со знакопеременной кривизной. Конечно, изучение внутренней геометрии конкретного многообразия знакопеременной кривизны или конкретной поверхности знакопеременной кривизны не вызывает принципиальных затруднений, однако неясно, как выделить общие свойства подобных объектов, которые и могли бы составить предмет содержательной теории.

К отмеченной задаче можно подойти, рассматривая многообразия со случайной кривизной. Так существует ряд теорем, описьвающих свойства близких геодезических со случайной кривизной, принимающей как положительные, так и отрицательные значения [3]. Можно показать, что близкие геодезические на таких многообразиях экспоненциально разбегаются, а поле Якоби вдоль геодезической обращается в нуль в бесконечной последовательности точек. Оба перечисленных свойства справедливы с вероятностью 1.

Таким образом, многообразия со случайной кривизной обнаруживают общность свойств как с многообразиями отрицательной, так и с многообразиями положительной кривизны. Поля Якоби на таких многообразиях демонстрируют экспоненциальньй рост абсолютной величины, сопровождаемый случайньми осциллящиями.

В работе [3] основной акцент сделан на изучении скорости роста поля Якоби; при этом описание поведения последовательности сопряженных точек остается за рамками

Работа выполнена при поддержке фонда INTAS, грант № 03-51-5807. 
исследования. В настоящей работе приводится построение оценки для среднего расстояния между соседними сопряженными точками.

Отметим, что как в работе [3], так и в настоящей работе случайная кривизна задается не на всем многообразии, а локально вдоль некоторой геодезической. О возможности построения вероятностных моделей, задающих кривизну на всем многообразии, см. [4].

2. Уравнение Якоби и случайная кривизна. Рассмотрим на двумерном римановом многообразии $M^{2}$ семейство геодезических $\gamma(\theta, x)$, пересекающихся в заданной точке $P$. При этом $\theta$ - угол, отсчитьваемый в точке $P$ от некоторой геодезической $\Gamma$ этого семейства, а $x$ - длина вдоль геодезических. Тогда полем Якоби $y$ в точке $x$, или геодезическим отклонением вдоль геодезической Г, называется величина

$$
y(x)=\left.\frac{\partial \gamma}{\partial \theta}\right|_{\theta=0} .
$$

Поле Якоби можно найти из уравнения Якоби (см. [1]), которое также назьвают уравнением геодезических отклонений:

$$
y^{\prime \prime}+k(x) y=0
$$

где $k$ - кривизна риманова пространства. Начальные условия для уравнения (2) имеют вид $y(0)=0$ и $y^{\prime}(0)=1$.

В дальнейшем мы будем считать кривизну $k(x)$ при $x>0$ случайным процессом. Предположим далее, что полуось $[0, \infty)$ значений $x$ разделена на равные отрезки точками $x_{0}=0, x_{1}=\delta, \ldots, x_{n}=n \delta, \ldots$, причем значения $k(x)=k_{n}(x)$ при $x_{n-1} \leqslant x<x_{n}$ статистически независимы между собой. Точки $x_{n}$ мы будем назьвать точками обновления.

Мы будем считать, что значения кривизны между точками обновления постоянно: $k(x) \equiv k_{n}$ при $x_{n-1} \leqslant x \leqslant x_{n}$. Таким образом, случайньй процесс $k(x)$ сводится к последовательности независимых одинаково распределенных случайных величин $\left\{k_{n}\right\}$.

При этих предположениях не вызывает сомнения, что для каждой реализации случайного процесса $k(x)$ уравнение $(2)$ с нашими начальными условиями имеет решение, которое на интервалах между точками обновления имеет непрерывные вторые производные. Отметим, что зная поле Якоби $y(x)$, можно восстановить и метрику (первую квадратичную форму) риманова пространства на геодезической Г. Эта метрика имеет вид

$$
d s^{2}=d x^{2}+y^{2}(x) d \theta^{2} .
$$

3. Исследование уравнения Якоби. Поскольку ниже будет рассматриваться асимптотическое приближение, уравнение Якоби следует обезразмерить. С этой целью введем безразмерную величину $t=x / \delta$, характеризующую количество моментов обновления на отрезке $[0 ; x]$. Безразмерное уравнение Якоби полностью сохранит свой вид

$$
y^{\prime \prime}+\nu(t) y=0
$$

где $\nu(t)=k(t) \delta^{2}$. Имея в виду совпадение уравнений (2) и $(4)$, величину $\nu$ естественно назвать безразмерной кривизной. Значения случайного процесса $\nu(t)$ теперь обновляются в целочисленных точках $t=1,2, \ldots$. Начальные условия для уравнения (4) имеют вид $y(0)=0$ и $y^{\prime}(0)=1$. 
Сделаем еще одну замену переменной:

$$
u(t)=\frac{y^{\prime}(t)}{y(t)}
$$

в результате которой порядок уравнения (4) понизится:

$$
u^{\prime}=-\nu(t)-u^{2}
$$

а начальное условие примет вид $u(0)=\infty$. Условие возникновения сопряженной точки $t^{*}$, т.е. точки, в которой $y=0$, в терминах $u$ формулируется как $u\left(t^{*}\right)=-\infty$.

Теперь сформулируем асимптотическое приближение, в котором будет проводиться все дальнейшее исследование. Будем считать, что имеется параметрическое семейство случайных процессов $\nu(t)=\nu(t, \varepsilon)$, каждый из которых обновляется в целочисленных точках. Будем считать безразмерную кривизну малой, устремив $\varepsilon \rightarrow 0$.

Для простоты дальнейшего изложения потребуем равномерной ограниченности значений процессов $\nu(t, \varepsilon)$ :

$$
|\nu(t, \varepsilon)| \leqslant \varepsilon .
$$

Указанные условия означают равномерную сходимость семейства процессов к нулевому процессу. В работе в качестве сходимости всегда будет использоваться равномерная сходимость, поэтому ниже условие $\varepsilon \rightarrow 0$ будет опускаться. Везде, где это не будет приводить к путанице, параметр $\varepsilon$ у случайных величин семейства $\nu_{n}(\varepsilon)$ также будет опущен.

В условиях равномерной ограниченности общую картину эволюции процесса $u(t)$ можно проследить исходя только из общего вида уравнения (5). Процесс стартует из точки $u_{0}=\infty$. Далее под воздействием члена $-u(t)^{2}$ значения $u(t)$ убывают вплоть до величины $\sqrt{\varepsilon}$. На интервале $(-\sqrt{\varepsilon} ; \sqrt{\varepsilon})$ происходит некоторое случайное блуждание, во время которого процесс $u(t)$ не может вьцити за верхнюю границу. Далее в некоторьй момент процесс пересекает нижнюю границу, и после этого его значения убывают вплоть до $-\infty$. В этот момент на геодезической образуется сопряженная точка. Как будет показано ниже, бесконечные интервалы $(\infty ; \sqrt{\varepsilon})$ и $(-\sqrt{\varepsilon} ;-\infty)$ преодолеваются процессом $u(t)$ за конечное с вероятностью 1 время.

Введем три марковских момента:

$$
\widehat{l}:=\inf _{t}\{t: u(t) \geqslant \sqrt{\varepsilon}\}, \quad \widehat{m}:=\inf _{t}\{t: u(t) \geqslant \sqrt{-\varepsilon}\}, \quad \widehat{n}:=\inf _{t}\{t: u(t)=-\infty\} .
$$

Как следует из определения, $\widehat{l}$ - момент первого достижения зоны случайного блуждания, $\widehat{m}$ - момент первого выхода из этой зоны, а $\widehat{n}$ - момент образования первой сопряженной точки.

Покажем, что справедлива следующая лемма.

ЛЕмма 1. Пусть выполнено условие равномерной ограниченности. Пусть далее существует $a>0$ такое, что $\mathrm{P}\{\nu(t, \varepsilon) \geqslant 0\}>$ а для всех $\varepsilon$. Тогда справедливь следующие оченки:

$$
\mathrm{E}(\widehat{l})=O_{p}\left(\frac{\ln (1 / \sqrt{\varepsilon})}{\sqrt{\varepsilon}}\right), \quad \mathrm{E}(\widehat{n}-\widehat{m})=O_{p}\left(\frac{\ln (1 / \sqrt{\varepsilon})}{\sqrt{\varepsilon}}\right) .
$$


ДокАЗАтЕльство. Рассмотрим вспомогательньй процесс $u_{0}$, определяемый системой уравнений

$$
u_{0}^{\prime}=\varepsilon-u_{1}^{2}, \quad u_{0}(0)=\infty .
$$

Эта система имеет следуюшее решение:

$$
t=\frac{1}{2 \sqrt{\varepsilon}} \ln \left|\frac{u_{0}+\sqrt{\varepsilon}}{u_{0}-\sqrt{\varepsilon}}\right| .
$$

Из решения видно, что процесс $u_{0}$ будет идти до точки $\sqrt{\varepsilon}$ бесконечное время. Однако любое другое значение большее, чем $\sqrt{\varepsilon}$, будет достигнуто за конечное время. В частности, значение $\sqrt{\varepsilon}+\varepsilon$ будет достигнуто за время

$$
O\left(\frac{\ln (1 / \sqrt{\varepsilon})}{\sqrt{\varepsilon}}\right) .
$$

Благодоря равомерной ограниченности (6) процесс $u_{0}(t)$ мажорирует исходный процесс $u(t)$. Следовательно, $u(t)$ достигнет значения $\sqrt{\varepsilon}+\varepsilon$ за время, не превосходящее $(9)$.

Из уравнения (5) видно, что процесс $u(t)$ преодолеет отрезок $[\sqrt{\varepsilon} ; \sqrt{\varepsilon}+\varepsilon]$ сразу же, как только значение $\nu(t, \varepsilon)$ станет больше 0 . Поскольку $\mathrm{P}\{\nu(t, \varepsilon) \geqslant 0\}>a$, это событие случится за среднее время, не превосходящее $1 / a$. Из этого следует первое утверждение леммы. Второе утверждение доказьвается аналогично.

Лемма 1 дает оценку среднего времени нахождения процесса вне зоны случайного блуждания $|u(t)|>\sqrt{\varepsilon}$. Теперь перейдем к оценке времени жизни процесса $u(t)$ в зоне случайного блуждания.

Рассмотрим вспомогательньй процесс $u_{1}(t)$, совпадающий с процессом $u(t)$ до входа в зону случайного блуждания и эволюционирующий под воздействием уравнения

$$
u_{1}^{\prime}=-\nu(t)
$$

в самой зоне случайного блуждания. Сравнение уравнений (5) и (10) показывает, что процесс $u_{1}(t)$ мажорирует исходньй процесс $u(t)$.

Процесс $\nu(t)$ обновляется в целочисленных точках. Следовательно, значения процесса $u_{1}(t)$, также взятые в целочисленных точках, образуют дискретное блуждание с независимыми равнораспределенньми приращениями.

Поскольку безразмерная кривизна бесконечно мала, дисперсия приращений процесса $u_{1}(t)$ стремится к нулю. Если дополнительно предположить, что среднее значение приращений равно нулю, то поведение такого случайного блуждания хорошо описьвается броуновским движением $w(t)$.

Подобно исходному процессу, процесс $u_{1}(t)$, попав в зону случайного блуждания, может сколь угодно близко подходить к верхней границе $\sqrt{\varepsilon}$, но не может ее пересечь. Можно сказать, что $u_{1}(t)$ как бы отражается от верхней границы зоны и снова начинает уменьшаться.

Данное рассуждение подсказьвает рассмотреть процесс $u_{*}(t)$, чье поведение в зоне случайного блуждания задается следующим образом:

$$
u_{*}(\widehat{l}+t)=\sqrt{\varepsilon}-\sqrt{\mathrm{D} \nu_{n}}|w(t)| .
$$

Для процесса $u_{*}(t)$ по аналогии с $(7)$ определим марковский момент $\widehat{m}_{*}$. Среднее значение этого момента можно оценить при помощи следующей леммы. 
Лемма 2. В условиях (7) и (11) справедливо следующее равенство:

$$
\mathrm{E}\left(\widehat{m}_{*}-\widehat{l}\right)=\frac{4}{\varepsilon \mathrm{D}\left(\nu_{n} / \varepsilon\right)} .
$$

ДокАЗАТЕЛЬство. Как следует из определения процесса $u_{*}(t)$, время $\widehat{m}_{*}-\widehat{l}$ равно времени, необходимому процессу $|w(t)|$ для того, чтобы достигнуть уровня $2 \sqrt{\varepsilon / \mathrm{D} \nu_{n}}$. По тождеству Вальда среднее значение этого времени равняется квадрату значения уровня, т.е. $4 \varepsilon / \mathrm{D} \nu_{n}$. Внеся $\varepsilon$ под знак дисперсии, получаем требуемое равенство. Лемма доказана.

Связь между интересуюшим нас моментом $\widehat{m}$ и уже оцененным моментом $\widehat{m}_{*}$ устанавливается следующей леммой.

ЛЕмма 3. Пусть выполнено условие равномерной ограниченности, $\mathrm{D}\left(\nu_{n} / \varepsilon\right)$ равномерно по в отделены от нуля, $а \mathrm{E} \nu_{n}=0$. Тогда

$$
\varepsilon \mathrm{E} \widehat{m} \leqslant \varepsilon \mathbf{E} \widehat{m}_{*}+o(1) .
$$

ДокАЗАТЕЛЬСТво. Подобно процессу $u_{1}(t)$ введем еще два процесса, совпадающих с процессом $u(t)$ до момента входа в зону случайного блуждания и несколько отличающихся в дальнейшем.

Процесс $u_{2}(t)$ эволюционирует под воздействием уравнения (10), но если он на каком-то целочисленном интервале становится больше, чем $\sqrt{\varepsilon}$, то вылезшая часть зеркально отражается обратно.

Процесс $u_{3}(t)$ эволюционирует под воздействием уравнения (5), но в те моменты времени, когда процесс $u_{2}(t)$ отражается, процесс $u_{3}(t)$ претерпевает разрьв и его значение полагается равным $\sqrt{\varepsilon}$. В такие моменты процесс $u_{3}(t)$ становится больше, чем процесс $u_{2}(t)$, но не более, чем на $\varepsilon$, потому как шаги процесса $u_{2}(t)$ не превосходят $\varepsilon$.

Для того, чтобы достичь нижнюю границу $-\sqrt{\varepsilon}$, процесс $u_{2}(t)$ должен в последний раз отразиться от верхней гранищы и отправиться вниз. По пути его непременно обгонит процесс $u_{3}(t)$, так как скорость последнего имеет дополнительную отрицательную добавку порядка $\varepsilon$. При этом количество шагов будет никак не менее $\sqrt{\varepsilon}$, а догнать нужно всего лишш разнищу в $\varepsilon$.

Из доказанного следует, что процесс $u_{2}$ достигнет значения $-\varepsilon$ позже, чем это сделает процесс $u_{3}$, а процесс $u_{3}$ позже, чем исходньй процесс $u$. Таким образом, в терминах марковских моментов имеем

$$
\mathrm{E} \widehat{m} \leqslant \mathrm{E} \widehat{m}_{2} .
$$

Согласно принципу инвариантности Донскера-Прохорова процесс $u_{2}(t)$ слабо сходится к процессу $u_{*}(t)$. Из слабой сходимости вытекает сходимость математических ожиданий случайных величин, зависящих от этих процессов. В частности,

$$
\varepsilon \mathrm{E} \widehat{m}_{2} \rightarrow \varepsilon \mathrm{E} \widehat{m}_{*} .
$$

Из равномерной отделенности от нуля $\mathrm{D}\left(\nu_{n} / \varepsilon\right)$ следует конечность $\varepsilon \mathrm{E} \widehat{m}_{*}$, поэтому осуществленньй предельный переход корректен.

Домножив обе части неравенства (13) на $\varepsilon$ и перейдя к пределу, получаем утверждение леммы. 
4. Основные результаты. Пусть $\widehat{x}_{n}$ - последовательность сопряженных точек. Центральный результат работы состоит в следующей теореме.

ТЕОрема 1. Пусть выполнено условие равномерной ограниченности, $\mathrm{E} \nu_{n}=0$, а величины $\mathrm{D}\left(\nu_{n} / \varepsilon\right)$ u $\mathrm{P}\left\{\nu_{n}(\varepsilon) \geqslant 0\right\}$ равномерно по в отделены от нуля. Тогда

$$
\mathrm{E}\left(\widehat{x}_{1}\right) \leqslant \frac{4 \delta}{\varepsilon \mathrm{D}\left(\nu_{n} / \varepsilon\right)}(1+o(1)) .
$$

ДокАЗАТЕЛЬСТво. В силу леммы 3 имеем

$$
\begin{aligned}
\varepsilon \mathrm{E} \widehat{n} & =\varepsilon \mathrm{E} \widehat{m}+\varepsilon \mathrm{E}(\widehat{n}-\widehat{m}) \leqslant \varepsilon \mathrm{E} \widehat{m}_{*}+\varepsilon \mathrm{E}(\widehat{n}-\widehat{m})+o(1) \\
& =\varepsilon \mathrm{E}\left(\widehat{m}_{*}-\widehat{l}\right)+\varepsilon \mathrm{E} \widehat{l}+\varepsilon \mathrm{E}(\widehat{n}-\widehat{m})+o(1) .
\end{aligned}
$$

Далее, подставляя оценки, полученные в леммах 1 и 2, имеем

$$
\varepsilon \mathrm{E} \widehat{n} \leqslant \frac{4}{\mathrm{D}\left(\nu_{n} / \varepsilon\right)}+o(1) .
$$

Разделив обе части неравенства на $\varepsilon$ и имея ввиду равномерную отделенность величин $\mathrm{D}\left(\nu_{n} / \varepsilon\right)$ от нуля, получаем

$$
\mathrm{E} \widehat{n} \leqslant \frac{4}{\varepsilon \mathrm{D}\left(\nu_{n} / \varepsilon\right)}(1+o(1)) .
$$

Полученное выражение дает оценку среднего числа отрезков обновления, лежащих до первой сопряженной точки. Умножив эту величину на расстояние между соседними точками обновления $\delta$, получаем требуемую оценку. Теорема доказана.

Теорема 1 дает оценку среднего расстояния до первой сопряженной точки. Оказывается, что точно такое же выражение применимо для оценки расстояния между двумя соседними точками.

Теорема 2. Пусть $\widehat{x}_{i}<\widehat{x}_{j}$ - некоторые сопряженные точки. В условиях теоремы 1 справедлива следующая асимптотическая оченка:

$$
\mathrm{E}\left(\widehat{x}_{j}-\widehat{x}_{i}\right) \leqslant \frac{4 \delta(j-i)}{\varepsilon \mathrm{D}\left(\nu_{n} / \varepsilon\right)}(1+o(1)) .
$$

ДокАЗАТЕЛьство. Пусть сопряженная точка $\widehat{x}_{i}$ принадлежит интервалу $\left[n^{*} \delta\right.$; $\left.\left(n^{*}+1\right) \delta\right]$. Расстояние $\widehat{x}_{i+1}-\widehat{x}_{i}$ с точностью до $\delta$ равно расстоянию до момента первого обращения в бесконечность процесса $u_{4}(t)=u\left(n^{*}+t\right)$. Этот процесс эволюционирует под воздействием уравнения

$$
u_{4}^{\prime}=-\nu\left(n^{*}+t\right)-u_{4}^{2}
$$

Поскольку в целочисленных точках процесс $\nu(t)$ обновляется, статистические свойства процессов $\nu(t)$ и $\nu\left(n^{*}+t\right)$ полностью идентичны. Поэтому идентичны уравнения $(5)$ и $(16)$, а следовательно, и законы эволюции процессов $u(t)$ и $u_{4}(t)$. Единственное отличие заключается в начальных условиях: $u(0)=\infty$, а $u_{4}(0) \leqslant \infty$. 
Это означает, что второй процесс в среднем быстрее попадет в зону случайного блуждания, однако среднее время, проводимое этими двумя процессами в самой зоне случайного блуждания, совпадает. Между тем, порядок расстояния между соседними сопряженньми точками определяется именно временем, которое процесс $u(t)$ тратит на преодоление зоны случайного блуждания. Поэтому среднее расстояние между двумя соседними сопряженными точками асимптотически равно среднему расстоянию до первой сопряженной точки. Умножив оценку (14) на $j-i$, получаем искомую оценку.

Автор благодарен В.Н.Тутубалину и Д. Д. Соколову за полезные обсуждения.

\section{СПИСОК ЦИТИРОВАННОЙ ЛИТЕРАТУРЫ}

[1] Громол Д., Клингенберг В., Мейер В. Риманова геометрия в целом. М.: Мир, 1971.

[2] Ефимов Н. В. Гиперболические задачи теории поверхностей // Тр. Международного конгресса математиков. М.: Мир, 1966.

[3] Ламбурт В.Г., Соколов Д. Д., Тутубалин В.Н. Поля Якоби вдоль геодезических со случайной кривизной // Матем. заметки. 2003. Т. 74. № 3. С. 416-424.

[4] Л амбурт В.Г., Розендорн Э.Р., Соколов Д. Д., Тутубалин В. Н. Геодезические со случайной кривизной на римановых и псевдоримановых многообразиях // Тр. геометр. семинара Казанского гос. ун-та. 2003. Т. 24. С. 99-106.

Московский государственный университет им. М.В. Ломоносова

Поступило

E-mail: lamburt@yandex.ru

28.07.2003

Исправленный вариант

05.04 .2005 